\title{
El Interés en la Química General para Ingenierías y el Bajo Rendimiento Escolar
}

\author{
Cesar G. Iñiguez-Monroy ${ }^{(1)^{*}}$, Wendolyn E. Aguilar-Salinas ${ }^{(1)}$, Maximiliano de las Fuentes-Lara ${ }^{(1)}$ y \\ Rosario E. Rodríguez-González ${ }^{(2)}$ \\ (1) Facultad de Ingeniería Mexicali, Universidad Autónoma de Baja California (UABC). Blvd. Benito Juárez \\ S/N, C.P. 21280. Mexicali, B.C., México (e-mail: cesar.iiguez@uabc.edu.mx, \\ aguilar.wendolyn@uabc.edu.mx,maximilianofuentes@uabc.edu.mx). \\ (2) Instituto de Ciencias Agrícolas, Universidad Autónoma de Baja California (UABC). Carretera a Delta \\ S/N, C.P. 21705. Ejido Nuevo León, B.C., México (e-mail: esmeralda.rodriguez@uabc.edu.mx).
}

${ }^{*}$ Autor a quien debe ser dirigida la correspondencia.

Recibido Dic. 7, 2016; Aceptado Feb. 3, 2017; Versión final Mar. 30, 2017, Publicado Ago. 2017

\begin{abstract}
Resumen
Para explorar las causas del aumento en el índice de reprobación de la materia de química general que se imparte en la Facultad de Ingeniería Mexicali, de la Universidad Autónoma de Baja California (UABC), se analizaron las actas de calificaciones del periodo 2011-2015 con modelos de tendencia lineal. Este índice ha incrementado 10\% en los últimos años. Durante el periodo 2014-2 al 2015-2 se aplicaron encuestas a estudiantes y sus respuestas se compararon con sus calificaciones mediante pruebas ANOVA unidireccionales. Los resultados mostraron un incremento en el porcentaje de estudiantes que perdieron derecho a examen ordinario o no lo presentaron. Las respuestas a preguntas relacionadas con la utilidad de la química mostraron una relación importante con sus calificaciones, las cuales fueron mayores para quienes si la consideran una ciencia útil. Por otro lado, la realización de prácticas de laboratorio durante el bachillerato también mostró un efecto positivo en dichas notas.
\end{abstract}

Palabras clave: química general; motivación escolar, índice de reprobación; rendimiento escolar

\section{Student's Interest in General Chemistry Course for Engineers and Low Learning Achievement}

\begin{abstract}
To explore the causes of the increasing of the failure rates in a general chemistry course delivered to students of the Engineering Faculty of Autonomous University of Baja California (UABC), in Mexicali, data gathered from teachers' final grade reports from 2011 to 2015 were analyzed with linear tendency models. This index has shown an increase of around $10 \%$ over the last years. A survey was applied to students during the 2014-2 to 2015-2 semesters, and the answers were compared to their grades by means of oneway ANOVA tests. The results showed that the percentage of students that missed the final exam, or had more than $20 \%$ absences during the course, increased on this period following a linear model. Students' answers to questions related to their interest for chemistry showed that the perception of usefulness of chemistry in their careers is strongly related with their final grades. On the other hand the fulfillment of laboratory practices during high school showed a positive effect on general chemistry grades.
\end{abstract}

Keywords: general chemistry; academic motivation; failure rate; learning achievement 


\section{INTRODUCCIÓN}

La enseñanza de la asignatura de química general, ubicada en el marco del tronco común universitario para las ingenierías, presenta retos particulares. Uno de estos retos está relacionado con el hecho de que esta materia es parte del primer semestre de las carreras universitarias de ingeniería, en donde los estudiantes se enfrentan a un nuevo y desconocido ambiente con el que aún no se sienten identificados (Ezcurra, 2004). Al no sentir pertenencia al programa educativo de su elección, se cuestionan acerca de la relevancia que tendrán los conocimientos de química general para el resto de sus carreras (Osorio García et al., 2009).

Dado que esta materia es precisamente la iniciación de los estudiantes universitarios con la ciencia (Talanquer, 2004); la forma en cómo los profesores abordamos su enseñanza, la forma en la que nuestros estudiantes la entienden, resulta de vital importancia para lograr despertar en ellos la curiosidad científica que los lleve a buscar respuestas, en contraposición con la aceptación popular de los fenómenos sin la necesidad de entenderlos. La química es una ciencia difícil de explicar, ya que es necesario recurrir a la estructura submicroscópica para explicar propiedades macroscópicas (Tejada et al., 2015).

Algunos autores han demostrado la importancia de los conocimientos previos adquiridos durante el nivel medio superior ( $\mathrm{Cu}$ Balán, 2005). Desde este punto de vista, la percepción del estudiante sobre la asignatura de química podría llegar a tener un sesgo negativo, dependiendo de su experiencia particular (Reyes y Obaya, 2008). La forma como se trabaja en el laboratorio tiene también un impacto en las notas finales y el nivel de confianza de los estudiantes con los conocimientos adquiridos (Muñoz-Osuna et al., 2014). El trabajo en el laboratorio permite adicionalmente desarrollar otras competencias como la del trabajo en equipo, que le ayuda al estudiante a analizar problemas con mayor y mejor criterio (Ramírez et al., 2013).

Se sabe que la deficiencia en los hábitos de estudio es un factor importante en las notas finales obtenidas por los estudiantes (Morales et al., 2009), aunque aún es necesario explorar la relación entre la motivación o simpatía que genera la asignatura con tales hábitos de estudio (Díaz et al., 2016). Otras dificultades reportadas mencionan la dificultad de incluir los conocimientos básicos de química en un solo semestre, que sean útiles a los estudiantes para poder entender asignaturas futuras específicas para sus carreras (Gómez-Moliné et al., 2008); o bien para que a partir de tales conocimientos el alumno sea capaz de entender problemas de la vida cotidiana y el desempeño profesional, y desarrolle una curiosidad científica (Izquierdo, 2004).

La actitud de los estudiantes en el aprendizaje de la química podría tener un efecto similar al que tiene la actitud en el aprendizaje de las matemáticas (Castañeda y Álvarez, 2004), en donde ha sido demostrada la existencia de una correlación significativa con las notas obtenidas. Adicionalmente las ideas preconcebidas acerca de la dificultad para aprender química, adquiridas durante las etapas escolares previas; constituyen un obstáculo para la continuación del aprendizaje en la universidad (Garriz et al., 2008). Las etapas previas de aprendizaje pueden sin embargo generar una curiosidad por el aprendizaje científico, la cual cambia la perspectiva de los estudiantes universitarios (Villalobos et al., 2016). Algunos investigadores han encontrado que además de problemas como la falta de madurez intelectual, falta de hábitos de estudio y motivación; algunos estudiantes muestran inseguridad y temor a manipular reactivos en el laboratorio de química, en el primer año universitario (Fiad y Galarza, 2015).

Los estudiantes que muestran poco interés en asignaturas como la química constituyen un grupo de riesgo de bajo aprovechamiento y reprobación (Chaparro et al., 2016). La detección oportuna, así como el establecimiento de estrategias dirigidas a estos grupos puede cambiar de forma significativa las expectativas de éxito de estos estudiantes no solamente en la asignatura en cuestión, sino en el resto de sus carreras (Ye et al., 2016).

El objetivo de este estudio fue analizar la relación entre el incremento en los índices de reprobación en la asignatura de química general, y factores como el interés de los estudiantes en dicha asignatura, su carrera de interés, y las competencias adquiridas durante la etapa media superior. Tales factores fueron relacionados con la variable calificación final en periodo ordinario El estudio fue llevado a cabo sobre la población de estudiantes de química general, perteneciente al programa de Tronco Común en Ciencias Básicas de la Ingeniería en la Facultad de Ingeniería Campus Mexicali, de la Universidad Autónoma de Baja California.

\section{MATERIALES Y MÉTODOS}

La metodología se presenta en dos subsecciones: en una se describen las características de la muestra y las preguntas realizadas a los estudiantes; y en la otra se describe la prueba estadística empleada. 


\section{Definición del universo de la población}

La información de los índices de reprobación fue tomada de las bases de datos de la institución educativa, con registros desde el año 2011. Las calificaciones reprobatorias reportadas como 'no presentó examen ordinario' (NP) y 'sin derecho a presentar examen ordinario' (SD), fueron analizadas por separado para evaluar su impacto en los índices mencionados. La nota SD se asigna generalmente por no cumplir con el $80 \%$ de asistencia Estos registros provienen de un total de 18 grupos con una población que oscila entre 30 y 40 estudiantes; los cuales fueron atendidos por 12 profesores diferentes, en horarios desde las 7 a.m. hasta las 9 p.m.

El contenido en la carta descriptiva de ésta materia es el mismo para toda la población estudiantil, independientemente del programa educativo que elijan, entre bioingeniería, ingeniería en energías renovables, aeroespacial, industrial, civil, mecánica, electrónica, eléctrica, computación o mecatrónica. El número de materias que requieren de conocimientos previos de química en cada carrera es diverso, siendo las carreras de bioingeniería e ingeniería en energías renovables las que tienen un mayor contenido de temas de química en sus planes de estudio (http://ingenieria.mxl.uabc.mx/).

Adicionalmente fueron aplicadas encuestas a estudiantes inscritos a la asignatura de Química General, al final de los periodos escolares 2014-2, 2015-1 y 2015-2; a un total de 405 estudiantes, la cual fue utilizada como muestra aleatoria para realizar inferencias estadísticas. Esta asignatura está ubicada en el primer semestre del Tronco Común en Ciencias de la Ingeniería. Las preguntas incluidas en la encuesta fueron las siguientes: (i) ¿Qué ingeniería piensa estudiar?; (ii) ¿Llevó laboratorio de química en la preparatoria? ; (iii) ¿Cree que la materia de química le servirá en su futura carrera?; y iv) ¿Lleva la materia de química por primera o segunda vez?

\section{Estudio estadístico}

Los datos de índice de reprobación fueron graficados en series de tiempo con un modelo de tendencia lineal, separados en sus tres componentes: \%SD, \%NP, o bien \% por nota reprobatoria. Las respuestas de las encuestas fueron relacionadas con la calificación final obtenida en periodo ordinario de cada estudiante, la cual se reporta en una escala numérica de 0 a 100 puntos. La primera pregunta tiene 10 niveles posibles por las carreras que se imparten en ésta facultad, mientras que el resto son dicotómicas: la dos y la tres con los niveles 'si' y 'no' y la cuatro con los niveles ' $1^{\mathrm{a}}$ ' $\mathrm{y}$ ' $\mathrm{2}^{\mathrm{a}}$.' Los datos fueron analizados en el software estadístico Minitab, utilizando un nivel de confianza del 95\%.

Con el fin de evaluar su efecto cualitativo, cada pregunta fue tratada como una variable independiente, mientras que la calificación fue tratada como la variable dependiente. Cada variable independiente fue comparada con las calificaciones de forma separada con una prueba ANOVA unidireccional (Cárcamo Oyarzún, 2012). Adicionalmente fueron obtenidos los valores promedio de las calificaciones para los dos niveles de la variable '¿cree que la materia de química le servirá en su futura carrera?' de forma separada, para cada uno de los niveles de las otras dos variables dicotómicas, respectivamente.

\section{RESULTADOS Y DISCUSIÓN}

Un análisis de tendencia de los datos del índice de reprobación con respecto al periodo semestral, obtenido mediante la aplicación de un modelo lineal, mostró que la mayor causa en el incremento en el tiempo de los índices de reprobación en la materia de química general es precisamente el ausentismo; representado por los estudiantes con una nota de NP o SD en las figuras 1 y 2 . El porcentaje de estudiantes que obtuvieron una nota reprobatoria, se mantuvo relativamente constante en el modelo de tendencia lineal, aun cuando los datos muestran un ascenso y un descenso alternado.

El índice de reprobación es típicamente más bajo en ésta materia, aunque no de forma exclusiva, en el segundo ciclo escolar del año, que comienza en el mes de agosto. Los estudiantes admitidos a la universidad en éste segundo ciclo son generalmente aquellos que obtuvieron un mayor puntaje en las pruebas de admisión. Aquellos que no logran ingresar en el segundo ciclo, deben esperar al primer ciclo del siguiente año que comienza en el mes de febrero. Éstas diferencias han sido discutidas por Chaparro et al (2016), quienes atribuyen a éste grupo de estudiantes bajos niveles socioeconómicos y de capital cultural, así como una organización familiar de poca implicación. A pesar de ello, los índices de reprobación en el periodo evaluado fueron constantes para el resto de las materias del primer semestre, incluyendo aquellas con índices mayores, como cálculo diferencial e integral y álgebra.

En el caso de los estudiantes sin derecho o que no presentaron examen, a pesar de la clara diferencia entre los ciclos escolares uno y dos, se ha encontrado una clara tendencia ascendente en el periodo evaluado. La 
figura 2 muestra una inconsistencia con el comportamiento intermitente mencionado, en el año 2013. Se cree que puede estar relacionado con inconsistencias en la asignación de la calificación, donde en algunos casos se podría haber asignado una calificación numérica reprobatoria en lugar de NP.

Aun cuando se desconoce si el porcentaje de estudiantes SD y NP ha aumentado en otras materias, si se sabe que en el resto de materias del primer semestre, el índice de reprobación se mantuvo relativamente constante en el periodo evaluado. Tomando en cuenta la complejidad del fenómeno del ausentismo, las preguntas realizadas a los estudiantes tuvieron el objetivo de analizar únicamente el interés de los mismos por la asignatura, en relación con la utilidad que esperan que la química tenga en sus carreras.

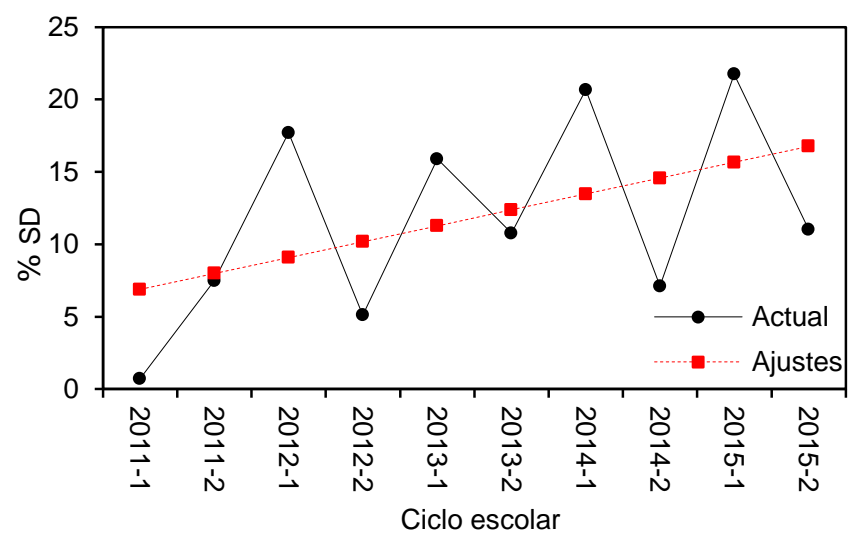

Fig. 1: Porcentaje de alumnos que se quedaron sin derecho a examen ordinario por no completar el $80 \%$ de asistencia (\%SD), en el periodo evaluado. Los puntos en rojo corresponden a un modelo de ajuste lineal.

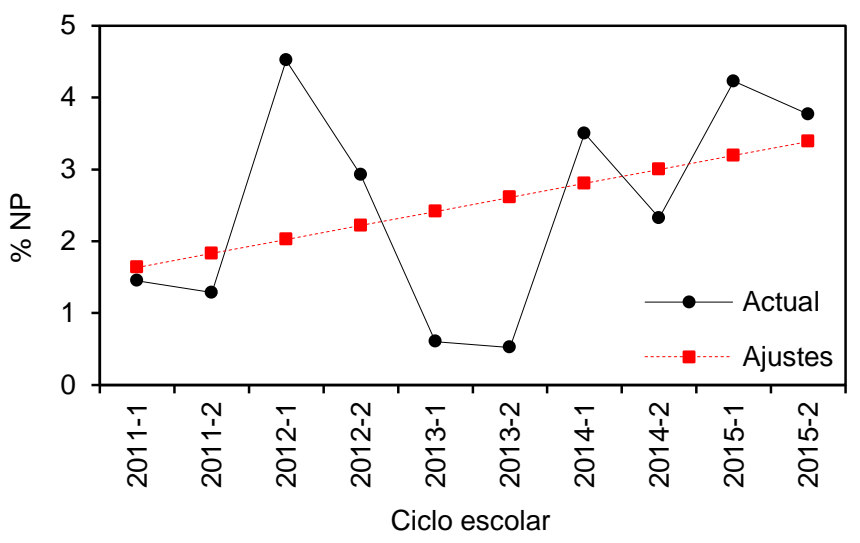

Fig. 2: Porcentaje de alumnos que no presentaron el examen ordinario (\%SD), en el periodo evaluado. Los puntos en rojo corresponden a un modelo de ajuste lineal.

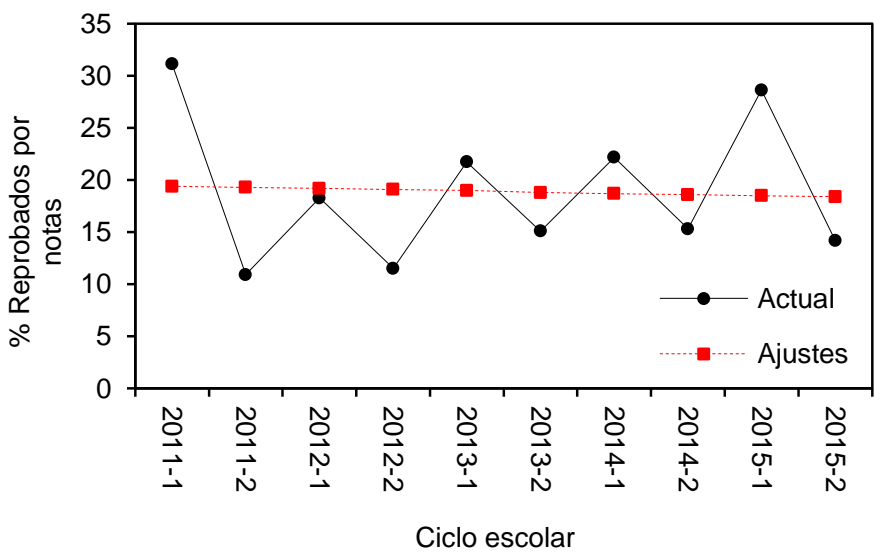

Fig. 3: Porcentaje de alumnos que obtuvieron nota reprobatoria en el periodo evaluado. Los puntos en rojo corresponden a un modelo de ajuste lineal.

La tabla 1 presenta los resultados de las calificaciones de los estudiantes de acuerdo a sus respuestas a las variables dicotómicas. La diferencia en el promedio de calificaciones para la percepción de utilidad de la 
química es de 10.8 puntos entre el nivel 'si' y el nivel 'no', que corresponde a un decremento del $19.1 \%$. La diferencia entre el 'si' y el 'no' para la pregunta ¿llevó laboratorio de química en la preparatoria?, es de 8.19 puntos (14.2\%). La diferencia entre el nivel ' $1^{a}$ vez' y el nivel ' $2^{a}$ vez' de la pregunta ¿lleva la materia de química por primera o segunda vez?, es de 15.9 puntos, que corresponde a un decremento del $29 \%$.

Tabla 1: Valores promedio e intervalo de confianza de las calificaciones para ambos niveles de las variables dicotómicas.

\begin{tabular}{|c|c|c|c|}
\hline & Nivel & $\begin{array}{c}\text { Calificación } \\
\text { Promedio }\end{array}$ & $\begin{array}{c}\text { Intervalo de } \\
\text { Confianza }\end{array}$ \\
\hline \multirow{2}{*}{ ¿Llevó laboratorio de química en la preparatoria? } & Si & 57.5 & $(52.4,62.6)$ \\
\hline & No & 49.3 & $(43.3,55.3)$ \\
\hline \multirow{2}{*}{ ¿Cree que la materia de química le servirá en su futura carrera? } & $\mathrm{Si}$ & 56.4 & $(52.0,60.8)$ \\
\hline & No & 45.6 & $(37.3,53.9)$ \\
\hline \multirow{2}{*}{ ¿Lleva la materia de química por primera o segunda vez? } & $1 \mathrm{a}$ & 54.8 & $(50.8,58.8)$ \\
\hline & $2 a$ & 38.9 & $(23.1,54.7)$ \\
\hline
\end{tabular}

La diferencia de 10.8 puntos entre ambos niveles de la variable 'utilidad de la química' es significativa para el nivel de confianza de la prueba (tabla 2). Estos resultados muestran por un lado la importancia del convencimiento personal de los estudiantes en la utilidad o el impacto que podría tener ésta asignatura en sus carreras, y sugiere que al ingresar a la universidad no han adquirido la competencia que les permita reconocerla (Fiad y Galarza, 2015). La necesidad constante de incrementar la cantidad de alumnos admitidos a la universidad podría generar un impacto negativo en los índices de abandono y reprobación si los estudiantes nuevos no tienen la habilidad de reconocer que existe un conjunto de competencias diversas que les permitirán desarrollarse como profesionales.

Ya que un reconocimiento negativo del impacto de la química está relacionada con un promedio inferior en las calificaciones, una vez que el estudiante toma la materia por segunda ocasión, el riesgo de abandonar la escuela crece, debido a que la reprobación de una asignatura por segunda ocasión provoca una situación administrativa de baja temporal, para la cual se requiere obtener la aprobación de un comité para tomarla por tercera ocasión, cuya reprobación causa baja definitiva.

Debido a que cada una de las carreras de ingeniería en ésta facultad tienen una cantidad diferente de materias con temas relacionados con la química general, la diferencia en la percepción de utilidad de ésta asignatura podría estar relacionada con la elección de la ingeniería. La figura 4 se muestra los resultados de un análisis categorizado por primera opción de carrera, los cuales muestran promedios de calificación significativamente bajos, inferiores al promedio general representado por la línea horizontal; tales como los que obtuvieron los estudiantes de ingeniería eléctrica, electrónica, industrial e ingeniería en computación.

Tabla 2: Resumen de las comparaciones ANOVA unidireccionales realizadas, mostrando la fuente de variación, grados de libertad (GL), suma de cuadrados (SC), media cuadrática (MC), cociente de las medias cuadráticas $(F)$ y el valor de probabilidad $(p)$.

\begin{tabular}{|c|c|c|c|c|c|}
\hline Fuente & $G L$ & $S C$ & $M C$ & $F$ & $P$ \\
\hline ¿Servirá en su carrera? & 1 & 6419 & 6419 & 4.56 & 0.033 \\
\hline Error & 354 & 498041 & 1407 & & \\
\hline Total & 355 & 504459 & & & \\
\hline Fuente & $G L$ & $S C$ & $M C$ & $F$ & $p$ \\
\hline ¿1a o 2a vez? & 1 & 3826 & 3826 & 2.68 & 0.102 \\
\hline Error & 358 & 510580 & 1426 & & \\
\hline Total & 359 & 514406 & & & \\
\hline Fuente & $G L$ & $S C$ & $M C$ & $F$ & $p$ \\
\hline ¿Lab en prepa? & 1 & 5829 & 5829 & 4.13 & 0.043 \\
\hline Error & 357 & 504382 & 1413 & & \\
\hline Total & 358 & 510211 & & & \\
\hline Fuente & $G L$ & $S C$ & $M C$ & $F$ & $p$ \\
\hline 1a opción de carrera & 9 & 1372 & 152 & 0.41 & 0.931 \\
\hline Error & 248 & 92826 & 374 & & \\
\hline Total & 257 & 94198 & & & \\
\hline
\end{tabular}


En contraste, los aspirantes a carreras como ingeniería aeroespacial, mecánica, bioingeniería, y energías renovables, obtuvieron calificaciones mayores al promedio. Estos resultados podrían sugerir una relación con el mayor contenido de química basados en los mapas curriculares de las ingenierías, aunque no especialmente con mecánica e ingeniería civil. Los resultados de la prueba ANOVA unidireccional (tabla 2), muestran que la diferencia en las calificaciones respecto a la carrera de elección no es significativa $(p>0.05)$.

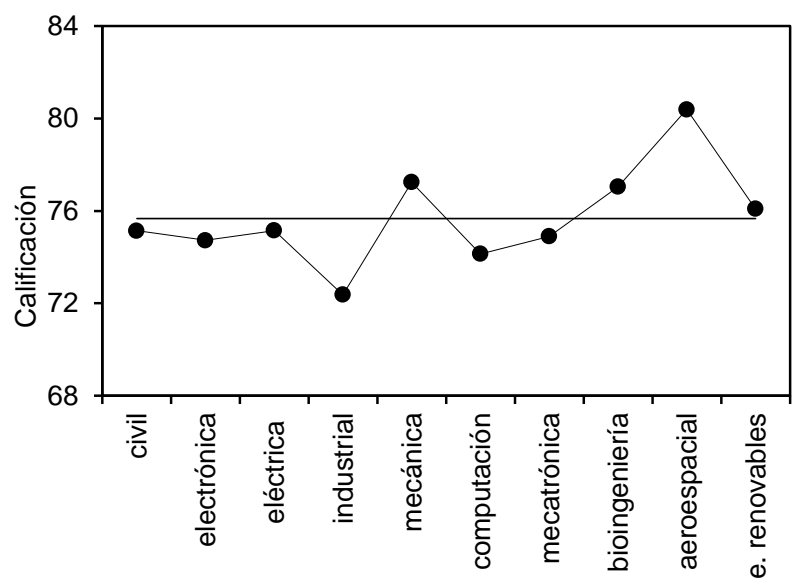

1a. opción de carrera

Fig. 4: Relación entre la primera opción de programa educativo de los estudiantes, y la calificación obtenida en periodo ordinario en la materia de química general. La línea horizontal representa el promedio global de los estudiantes que respondieron la pregunta.

A pesar de ello, la percepción de utilidad de la química sí tiene un efecto en las calificaciones categorizadas por primera opción de carrera, como se muestra en la figura 5. Las calificaciones obtenidas por los estudiantes que creen que la asignatura de química sí tendrá impacto en sus carreras, son consistentemente más altas que las de aquellos que creen lo contrario (figura 5), Todos los estudiantes que tienen como primera opción la bioingeniería declararon que la química sí será importante en sus carreras.

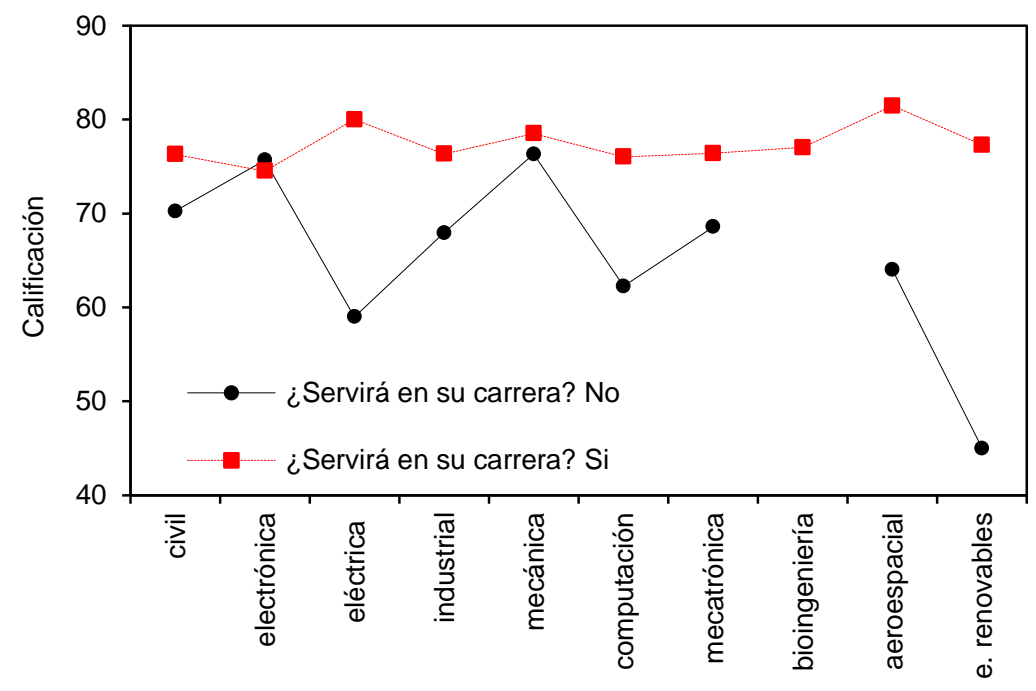

1a. opción de carrera

Fig. 5: Relación cualitativa entre los factores 'utilidad de la química en sus carreras' y 'primera opción de carrera' con respecto a la calificación obtenida por los estudiantes en periodo ordinario.

La comparación entre las respuestas a las preguntas de la encuesta, separadas con respecto a ambos niveles de la variable 'utilidad de la química' mostró tendencias adicionales. Por un lado, la diferencia en las calificaciones en periodo ordinario de los estudiantes que llevan la unidad de aprendizaje por primera o segunda ocasión es sensible a su percepción de utilidad de la química (tabla 3). En el caso de los estudiantes que estudian esta materia por primera vez, el promedio fue 78 para quienes piensan que la química es importante, frente a 69.7 para quienes piensan lo contrario. Esta diferencia corresponde a un decremento de $10.6 \%$. Por otro lado, la diferencia de calificación para los estudiantes que tomaron la materia por segunda ocasión fue de aproximadamente 30 puntos, que corresponde a un decremento de $45.3 \%$. 
Tabla 3: Comparación de las calificaciones para los niveles de las variables dicotómicas, con respecto a la percepción de utilidad de la química en las futuras carreras de los estudiantes encuestados.

\begin{tabular}{|c|c|c|c|}
\hline & & \multicolumn{2}{|c|}{$\begin{array}{c}\text { ¿Cree que la materia de química le servirá en } \\
\text { su futura carrera? }\end{array}$} \\
\hline & & $\mathrm{Si}$ & No \\
\hline & & \multicolumn{2}{|c|}{ Calificación } \\
\hline \multirow{2}{*}{$\begin{array}{l}\text { ¿Llevó laboratorio de química en la } \\
\text { preparatoria? }\end{array}$} & $\mathrm{Si}$ & 78.0 & 71.0 \\
\hline & No & 76.3 & 61.1 \\
\hline \multirow{2}{*}{$\begin{array}{l}\text { ¿Lleva la materia de química por } 1 \text { a } 02 a \\
\text { vez? }\end{array}$} & $1 \mathrm{a}$ & 78.0 & 69.7 \\
\hline & $2 a$ & 64.6 & 35.3 \\
\hline
\end{tabular}

De acuerdo a éstos resultados, existe un grupo de estudiantes especialmente vulnerable, constituido por aquellos que están repitiendo la materia, pero siguen sin considerarla útil. Estos estudiantes son los que podrían abandonar sus estudios, lo cual hace necesario desarrollar iniciativas para cambiar la percepción de utilidad de la química tanto en los estudiantes de primer ingreso como en aquellos que cursan por segunda ocasión. Finalmente, los resultados de las calificaciones obtenidas con respecto a la variable 'laboratorio en prepa' nos muestran que si existe una diferencia significativa en los promedios separados por los niveles de ésta variable dicotómica, de acuerdo a la prueba ANOVA unidireccional (tablas 1 y 2). La diferencia en las calificaciones promedio entre el grupo que si tuvo prácticas de química en el bachillerato y el que no tuvo fue de ocho puntos $(14.2 \%)$.

Aun cuando es necesario revisar otros factores que afectan las competencias adquiridas por los estudiantes en el bachillerato, éstos resultados sugieren que la percepción de utilidad de la química podría estar influenciada por la falta en la realización de prácticas de laboratorio en la etapa previa a la universidad; de forma que el estudiante no logra relacionar la materia con algo concreto (Martín et al, 2015). Del total de los estudiantes encuestados, el $40 \%$ declaró no haber realizado prácticas de química en el bachillerato (figura $6)$.

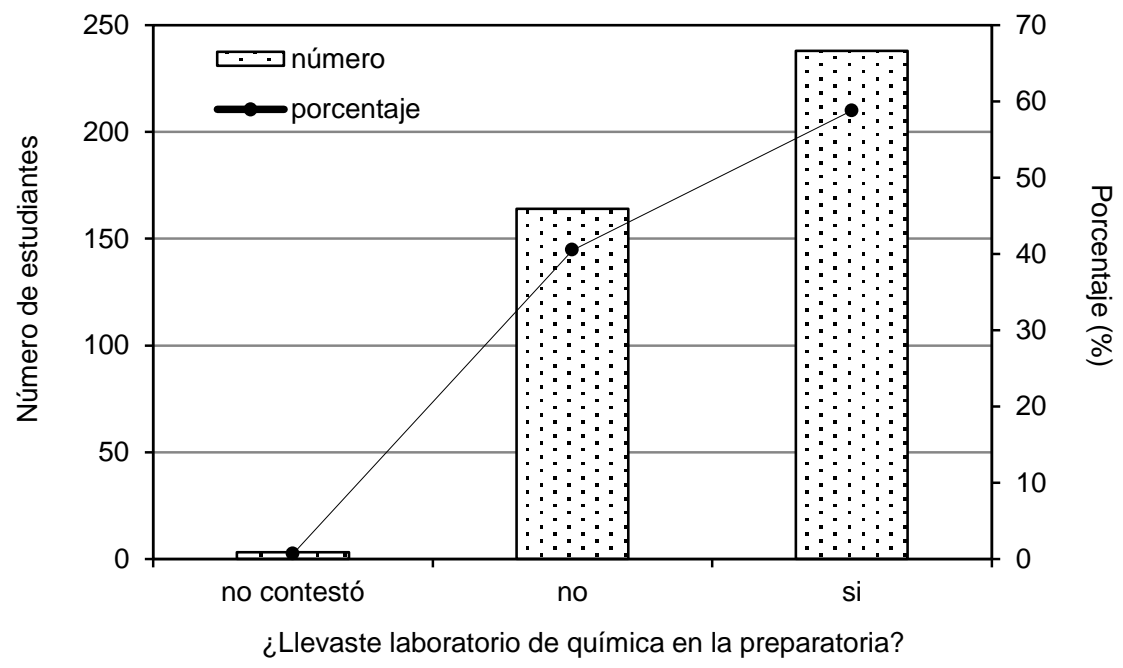

Fig. 6: Clasificación de estudiantes de acuerdo a su asistencia a prácticas de laboratorio en el bachillerato.

\section{DISCUSIÓN FINAL}

El análisis de tendencia lineal mostró que el porcentaje de estudiantes que se queda sin derecho a examen, así como el porcentaje de estudiantes que no presenta el examen ordinario de la asignatura de química general en el tronco común de ciencias de la ingeniería aumentó paulatinamente en el periodo evaluado. Los datos muestran una fluctuación periódica, donde los índices de reprobación para los estudiantes que ingresan en febrero son mayores, de 10-15\% para alumnos SD y de 7-10\% para alumnos reprobados por nota. Ésta diferencia podría atribuirse al nivel socioeconómico, capital cultural e implicación familiar, sugerida por Chaparro et al., 2015. Sin embargo es necesario estudiar ésta fluctuación para crear estrategias más efectivas que las actuales, limitadas a un periodo propedéutico de adaptación. 
Esta tendencia fue ligada con una pérdida de interés de los estudiantes por dicha unidad de aprendizaje, ya que en el periodo evaluado, los índices de reprobación del resto de las materias del primer semestre se mantuvieron relativamente constantes. Los estudiantes que no consideran que la química será importante en sus carreras obtuvieron en promedio 10/100 puntos menos en su calificación de ordinario, con respecto a aquellos que si la consideran importante. Estos resultados concuerdan con observaciones hechas por Fiad y Galarza (2015), en el sentido de que las estrategias de los estudiantes para alcanzar sus objetivos de aprendizaje son usadas en función de factores motivacionales.

Esta diferencia es consistente para los aspirantes a cada una de las carreras de esta facultad, demostrando el impacto que tiene la percepción que tienen sobre esta unidad de aprendizaje los estudiantes del tronco común. Desde este punto de vista resulta muy importante el papel del profesor de química general especialmente en las primeras sesiones, quien debe ser capaz de mostrar cómo puede ser útil la química en sus respectivas carreras; más aun considerando que los estudiantes que toman la asignatura por segunda ocasión en general siguen considerando que no es importante.

Sin embargo, el interés de los estudiantes universitarios en la química puede tener un antecedente en las etapas previas de su preparación. Un $40 \%$ de los estudiantes, quienes declararon no haber realizado prácticas de laboratorio en la etapa previa a la universidad; obtuvieron una calificación significativamente menor. Martín et al, (2015), resaltan la importancia de dar a los alumnos la oportunidad de contrastar con la medición experimental, siempre que sea posible, las teorías y datos transferidos como válidos en las clases teóricas.

Aun cuando no existen estudios que nos permitan conocer todos los factores que inciden en la falta de capacidad de los estudiantes para reconocer la utilidad de una ciencia como la química, en el paso del bachillerato a la universidad; si se han documentado acciones que pueden ayudar a generar una actitud positiva hacia los conceptos tratados en clase; como el uso de laboratorios virtuales (Fiad y Galarza, 2015). Estos autores han reportado en los alumnos inseguridad, desconfianza y temor a manipular reactivos y elementos básicos de un laboratorio. En éste sentido es recomendable aplicar éste tipo de prácticas innovadoras como una medida de control del problema en cuestión, así como la realización de estudios que nos permitan en vinculación con los niveles educativos previos, encontrar medidas de prevención.

\section{CONCLUSIONES}

De acuerdo al estudio y a los resultados presentados, se pueden obtener las siguientes conclusiones principales:

(1) La falta de conocimiento de los alcances de una materia como la química, no solamente en su relación con asignaturas posteriores, sino en el desempeño profesional; tiene como consecuencia un aprendizaje limitado, motivado por el deseo de aprobar y no de obtener un conocimiento útil; actitud que se mantiene a pesar de tener la necesidad de cursarla nuevamente. El grupo más vulnerable de estudiantes es el que cursa la materia por segunda oportunidad y no es capaz de reconocer la utilidad de ésta materia.

(2) Una de los factores ligados a la capacidad de reconocimiento de la utilidad de la química como una ciencia útil, es el de la realización de actividades prácticas de ésta materia en las etapas previas a la universidad.

(3) Es necesario incorporar capacitación a los profesores para darles herramientas que les permitan modificar dicha percepción negativa en los estudiantes, especialmente en las primeras semanas del curso, o bien como un recurso propedéutico. Esta iniciativa puede verse beneficiada del desarrollo y uso de contenidos innovadores, como los simuladores o laboratorios virtuales.

\section{REFERENCIAS}

Cárcamo Oyarzún, J. El profesor de Educación Física desde la perspectiva de los escolares, Estudios pedagógicos (Valdivia), 38(1), 105-119 (2012)

Castañeda, A. y Álvarez, M.J. La reprobación en matemáticas. Dos experiencias, Tiempo de Educar, 5(9), 141-172 (2004)

Chaparro, A., González, C. y Caso, J. Familia y rendimiento académico: configuración de perfiles estudiantiles en secundaria, Revista Electrónica de Investigación Educativa, 18(1), 53-68 (2016) 
Cu Balán, G. El impacto de la escuela de procedencia del nivel medio superior en el desempeño de los alumnos en el nivel universitario, Revista Electrónica Iberoamericana sobre Calidad, Eficacia y Cambio en la Educación, 3(1), 764-769 (2005)

Díaz, C., García, J. y Molina, A. ¿Dónde está la clave del éxito académico? Un análisis de la relación entre el uso del tiempo y el rendimiento académico, Cultura y Educación, 28(1), 173-195 (2016)

Ezcurra, A.M. Diagnóstico preliminar de las dificultades de los alumnos de primer ingreso a la educación superior, Perfiles Educativos, 23(107), 118-133 (2004)

Fiad, S.B. y Galarza, O.D. El Laboratorio Virtual como Estrategia para el Proceso de EnseñanzaAprendizaje del Concepto de Mol, Formación Universitaria, 8(4), 3-14 (2015)

Garriz, A., Nieto, E. Padilla, K., Reyes-Cárdenas, F. y Trinidad, R. Conocimiento didáctico del contenido en química. Lo que todo profesor debería poseer, Campo Abierto, 27(1), 153-177 (2008)

Gómez-Moliné, M., Morales, M.L. y Reyes-Sánchez, L.B. Obstáculos detectados en el aprendizaje de la nomenclatura química, Educación Química, 19(3), 201-206 (2008)

Izquierdo Aymerich, M. Un nuevo enfoque de la enseñanza de la química: contextualizar y modelizar, The Journal of the Argentine Society, 92(4/6), 115-136 (2004)

Martín, E., Fernández, E. y Atienza, J. La Docencia en Ciencias Medioambientales en la Universidad Autónoma de Madrid: Teoría y Práctica, Formación Universitaria, 8(6), 3-12 (2015)

Morales Burgos, A., García Sosa, J. y Escalante Triay, E. Causas de reprobación en los cursos de Mecánica de Fluidos de la Facultad de Ingeniería de la Universidad Autónoma de Yucatán, Ingeniería, Revista Académica, 13(3), 45-51 (2009)

Muñoz-Osuna, F.O., Arvayo-Mata, K.L., Villegas-Osuna, C.A., González-Gutiérrez, F.H., Sosa-Pérez, E.O. El método colaborativo como una alternativa en el trabajo experimental de Química Orgánica, Educación Química, 24(4), 464-469 (2014)

Osorio García, M., Mejía Serafín, L., Navarro Zavaleta, J.A. Factores psicosociales que influyen en el éxito o fracaso del aprovechamiento escolar en la asignatura de Física básica. Caso del plantel Ignacio Ramírez Calzada, Espacios Públicos, 12(26), 261-276 (2009)

Ramírez, J.A., Alejo, M.G., Jiménez, R. y Marmolejo, S. Percepción de los estudiantes de ciencias químicas sobre sus equipos de trabajo, Formación Universitaria, 6(3), 3-12 (2013)

Reyes, S.L. y Obaya, V.A. Hábitos de Estudio de Alumnos de Ingeniería Agrícola y su Impacto en el Rendimiento Obtenido en un Curso de Química Básica, Formación Universitaria, 1(5), 29-34 (2008)

Talanquer, V. Formación docente: ¿Qué conocimiento distingue a los buenos maestros de química?, Educación Química, 15(1), 60-66 (2004)

Tejada, C.N., Acevedo, D. y Gattas, C. Influencia de los Textos de Química en la Enseñanza y Aprendizaje del Concepto de Valencia, Formación Universitaria, 8(3), 27-34 (2015)

Villalobos, V., Ávila, J. y Olivares, S. Aprendizaje basado en problemas en química y el pensamiento crítico en secundaria, Revista Mexicana de Investigación Educativa, 21(69), 557-581 (2016)

Ye, L., Shuniak, C., Oueini, R., Robert, J. and Lewis, S. Can they succeed? Exploring at-risk students' study habits in college general chemistry, Chemistry Education Research and Practice, 17(4), 878-892 (2016) 
